\title{
Mitteilungen der Deutschen Schlaganfall-Gesellschaft
}

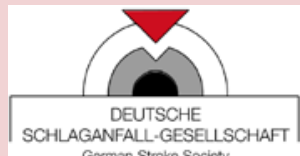

Nervenarzt 2011 $\cdot 82: 530$

DOI 10.1007/s00115-011-3294-7

C) Springer Medizin Verlag 2011

Redaktion

Prof. Dr. med. Otto Busse

busse@dsg-berlin.org

\section{Erstmals Tele-Stroke- Einheiten zertifiziert}

In Ergänzung zu den Zertifizierungen regionaler und überregionaler Stroke Units hat sich die Deutsche Schlaganfall-Gesellschaft (DSG) entschlossen, auch Tele-Stroke-Units innerhalb eines regionalen Netzwerks zu zertifizieren. Erstmals wurden im Januar 2011 Tele-Stroke-Units im STENO-Netzwerk (Schlaganfall-Netzwerk mit Telemedizin Nord-Bayern) einschließlich ihrer Beratungszentren in Erlangen, Bayreuth und Nürnberg begutachtet. Erfreulicherweise waren die von der DSG geforderten Kriterien zumeist erfüllt. In einzelnen Fällen, sowohl bei den Te-
le-Stroke-Einheiten als auch bei den Beratungszentren, bestand jedoch noch Nachbesserungsbedarf. Wie bei den Stroke-UnitZertifizierungen erfolgt die Begehung durch einen leitenden Auditor der LGA-InterCert und einen medizinischen Auditor der DSG. Diese beiden werden durch einen Experten aus dem Bereich der telemedizinischen Schlaganfallversorgung ergänzt.

In nächster Zukunft sind weitere Begehungen zur Zertifizierung der Tele-Stroke-Einheiten im STENO-Netzwerk und anderen bestehenden Netzwerken geplant.

\section{Kardiologen und Schlagan- fallmediziner tauschen sich über Chest Pain Units und Stroke Units aus}

Erstmalig haben sich Vertreter der Deutschen Gesellschaft für Kardiologie - Herz- und Kreislaufforschung (DGK) und der Deutschen Schlaganfall-Gesellschaft (DSG) am 9. Februar 2011 in der Geschäftsstelle der DGK in Düsseldorf zu einem Erfahrungsaustausch bezüglich der Chest Pain Units und der Stroke Units sowie ihrer Zertifizierung getroffen. Für die DSG nahmen O. Busse, J. Faiss, D. Nabavi und B. Ringelstein, für die DGK P. Kirchhoff, K. Papoutsis, F. Post und R. Zahn zu den Stroke Units. Die hin und wieder geäußerte Befürchtung, dass insbesondere in kleineren Häusern die Krankenhausleitung auf die Idee kommen könnte, beide Units zusammenzulegen, dürfte damit gegenstandslos sein. Vorreiter für die Chest Pain Units in kardiologischen Kliniken war das Universitätsklinikum Mainz, das durch große Kampagnen zur Sensibilisierung der Patienten beigetragen hat und in dieser Hinsicht Vorbildfunktion hat.

Die Teilnehmer der Sitzung stellten viele Parallelen im Zertifizierungsprozess beider Units fest. Die DSG hat ihr Verfahren jedoch bereits mehrfach geändert, die DGK noch nicht.
Alle Teilnehmer waren sich abschließend einig, dass man gegenseitig viel voneinander lernen könne - sowohl in der Entwicklung derartiger Units als auch bei den Zertifizierungsverfahren. Die Kardiologen zeigten sich dankbar für die langjährigen Erfahrungen mit der Zertifizierung von Stroke Units, von denen sie profitieren können. Ein ausführlicher Bericht über das Arbeitstreffen mit Darstellung der Unterschiede und Gemeinsamkeiten in der Struktur, den Prozessen und bei der Zertifizierung beider Einheiten ist vorgesehen.

\section{Festveranstaltung anlässlich des zehnjährigen Jubiläums der Deutschen Schlaganfall- Gesellschaft}

2011 feiert die Deutsche Schlaganfall-Gesellschaft (DSG) ihr zehnjähriges Bestehen. Seit 2001 engagiert sich die Fachgesellschaft dafür, Forschung, Versorgung und Fortbildung auf dem Gebiet der Schlaganfallmedizin voranzubringen. In den vergangenen Jahren wurde bereits viel erreicht - ein Beispiel hierfür ist der große Erfolg der zertifizierten Stroke Units in Deutschland.

Anlässlich ihres Jubiläums lädt die DSG ihre Mitglieder sowie interessierte Kollegen am 24. Mai 2011 von 15.00 bis 17.30 Uhr $\mathrm{zu}$ einer Festveranstaltung in das Congress Center Hamburg (Raum 8) ein. Die Jubiläumsveranstaltung der DSG findet im Rahmen der European Stroke Conference (ESC) statt.

Teilnehmer der European Stroke Conference 2011 können die Festveranstaltung der DSG kostenlos und ohne zusätzliche
Anmeldung besuchen. Informationen zur ESC-Registrierung finden sich unter www.eurostroke.eu. Interessierte, die ausschließlich an der DSG-Veranstaltung, nicht aber an der gesamten ESC teilnehmen wollen, schicken bitte eine E-Mail mit Ihrem Vor- und Nachnamen sowie kompletter Postadresse an Frau Andrea Blättler (esc@congrex.com). Ihr Namensschild, das Sie zur kostenfreien Teilnahme am DSG-Symposium berechtigt, wird dann vor Ort am Sekretariat für Sie zur Abholung bereitliegen. Bei Fragen zur Registrierung kontaktieren Sie bitte Andrea Blättler unter esc@congrex. com oder Tel. +41616867711.

\section{Prof. Dr. Joachim Röther} Erster Vorsitzender der DSG

\section{Prof. Dr. Otto Busse}

Generalsekretär der DSG 